\title{
Extensions to 2-factors in bipartite graphs
}

\author{
Jennifer Vandenbussche* Douglas B. West ${ }^{\dagger}$ \\ Submitted: Feb 16, 2012; Accepted: July 25, 2013; Published: Aug 2, 2013 \\ Dedicated to the memory of Radhika Ramamurthi
}

\begin{abstract}
A graph is $d$-bounded if its maximum degree is at most $d$. We apply the OreRyser Theorem on $f$-factors in bipartite graphs to obtain conditions for the extension of a 2-bounded subgraph to a 2-factor in a bipartite graph. As consequences, we prove that every matching in the 5 -dimensional hypercube extends to a 2 -factor, and we obtain conditions for this property in general regular bipartite graphs. For example, to show that every matching in a regular $n$-vertex bipartite graph extends to a 2 -factor, it suffices to show that all matchings with fewer than $n / 3$ edges extend to 2 -factors.
\end{abstract}

\section{Introduction}

Haggvist [10] posed the following general question: Given a subgraph $F$ consisting of disjoint paths in a graph $G$, what conditions on $G$ and $F$ guarantee that $F$ extends to a spanning cycle in $G$ ? The question was studied for the case where $F$ is a matching in [2] and [22]. Another approach has been to place connectivity constraints and/or degree-sum constraints on $F$ (see $[3,11,12,19])$. In general, a disjoint union of paths is a linear forest.

Haggvist's question has been studied also when $G$ is the $d$-dimensional hypercube $Q_{d}$. Dvořák [4] showed that if a linear forest $F$ in $Q_{d}$ has at most $2 d-3$ edges, then $F$ extends to a spanning cycle in $Q_{d}$. Ruskey and Savage [17] asked whether every matching in $Q_{d}$ extends to a spanning cycle. Fink [6,7] proved the conjecture of Kreweras [15] that the answer is yes when $F$ is a perfect matching (the question remains open for general matchings).

We ask whether a weaker conclusion holds. Let $G$ be a graph, and let $H$ be a spanning subgraph of $G$ whose components are paths and cycles. Edmonds and Johnson [5] defined a $b$-matching in $G$ to be a subgraph of $G$ in which every vertex has degree at most $b$; in

\footnotetext{
*Mathematics Department, Southern Polytechnic State University, Marietta GA, jvandenb@spsu.edu

${ }^{\dagger}$ Mathematics Departments, Zhejiang Normal University, Jinhua, China, and University of Illinois, Urbana IL, west@math.uiuc.edu. Research partially supported by the National Security Agency under Award No. H98230-10-1-0363.
} 
their terminology $H$ is a 2-matching. Since we are studying extensions of subgraphs to spanning subgraphs (factors) with specified properties, we define a d-bounded factor to be a spanning subgraph with maximum degree at most $d$; hence $H$ is a 2-bounded factor. We ask for conditions guaranteeing that a 2-bounded factor extends to a 2-factor of $G$, restricting to the case where $G$ is bipartite.

A vertex weight $f$ assigns nonnegative integers to the vertices of a graph $G$. Given a vertex weight $f$, an $f$-factor in a graph $G$ is a spanning subgraph $H$ such that $d_{H}(v)=f(v)$ for $v \in V(G)$. The Ore-Ryser Theorem gives a necessary and sufficient condition for the existence of an $f$-factor in a bipartite graph. This elegant result and its history are not sufficiently well known. It appears to have been proved first by Ore [16]. Katerinis [14] called it the Ore-Ryser Theorem, without providing a reference to Ryser's work. Later authors continued to call it the Ore-Ryser Theorem.

The simplest of several ways to state the theorem parallels Hall's Theorem for bipartite matching. We recall standard terminology and notation. The neighborhood of a vertex $x$ in a graph $G$ is denoted $N_{G}(x)$ or simply $N(x)$. Let $N_{A}(y)=N(y) \cap A$, and let $N_{G}(A)$ or simply $N(A)$ denote $\bigcup_{x \in A} N(x)-A$. An $(X, Y)$-bigraph is a bipartite graph with partite sets $X$ and $Y$. For a vertex weight $f$ and a set $S \subseteq V(G)$, let $f(S)=\sum_{v \in S} f(v)$.

Theorem 1. Let $G$ be an $(X, Y)$-bigraph. If $f$ is a vertex weight on $G$, then $G$ has an $f$-factor if and only if $f(X)=f(Y)$ and, for all $A \subseteq X$,

$$
f(A) \leqslant \sum_{y \in N(A)} \min \left\{f(y),\left|N_{A}(y)\right|\right\} .
$$

When $|X|=|Y|$ and $f(v)=1$ for all $v$, the condition reduces to Hall's Condition for a perfect matching in $G$. In general, the condition is obviously necessary. Sufficiency can be proved from Tutte's $f$-Factor Theorem. When studying bipartite graphs, however, we seek elementary direct proofs, in light of the relative simplicity of matchings and factors in bipartite graphs. One such proof of the Ore-Ryser Theorem appears in Tutte [20], where Tutte describes it as "Ore's Theorem". The outline of a proof using network flow appears in [9]. One can also deduce the Ore-Ryser Theorem from the theorem of Folkman and Fulkerson [8] on $(g, f)$-factors in bipartite graphs, an elementary proof of which appears in [13] and in [1]. Finally, the Ore-Ryser Theorem can also be proved inductively like Hall's Theorem.

Motivated by seeking regular bipartite graphs in which all matchings extend to 2factors, we begin by obtaining properties of a minimal violation for extension of 2-bounded factors. To extend a 2-bounded factor $H$ to a 2-factor of $G$, we need an $f_{H}$-factor in $G$, where $f_{H}(v)=2-d_{H}(v)$ for $v \in V(G)$. We seek conditions for a minimal 2-bounded factor $H$ and a minimal set $A$ such that the inequality in the Ore-Ryser Theorem for an $f_{H}$-factor in $G-E(H)$ fails at $A$. We do this first for a general 2-bounded factor $H$ in Section 2 and then obtain further conditions in Section 3 when $H$ is a matching.

If no $H$ and $A$ can satisfy these properties, then every matching in $G$ extends to a 2 -factor. From this we obtain some applications. In a small step toward the RuskeySavage question on extending matchings to spanning cycles, we prove in Section 4 that 
every matching in $Q_{5}$ extends to a 2-factor. Whether all matchings in larger hypercubes extend to 2-factors remains open.

For a general $k$-regular bipartite graph $G$, we show in Section 3 that "large" matchings need not be checked. For $k \in\{3,4\}$, it suffices to check that $M$ extends when $D_{M} \leqslant k-2$, where $D_{M}$ is the maximum degree of the subgraph of $G$ induced by the vertices of $M$. For a general $n$-vertex regular bipartite graph, it suffices to show that every matching with fewer than $n / 3$ edges extends.

\section{Applying the Ore-Ryser Theorem}

Let $G$ be a $d$-regular bipartite graph with bipartition $(X, Y)$, and let $H$ be a 2-bounded factor in $G$. Let $G^{\prime}=G-E(H)$. For $i \in\{0,1,2\}$, let $X_{i}=\left\{x \in X: d_{H}(x)=2-i\right\}$; that is, $X_{i}$ is the set of vertices in $X$ that need $i$ additional edges when extending $H$ to a 2-factor in $G$. Partition $Y$ into $\left\{Y_{0}, Y_{1}, Y_{2}\right\}$ similarly.

Definition 1. For $A \subseteq X$, the sets $A_{0}, A_{1}, A_{2}$ and $B_{0}, B_{1}, B_{2}$ associated with $A$ are defined by $A_{i}=A \cap X_{i}$ and $B_{i}=N(A) \cap Y_{i}$. In addition, partition $B_{2}$ into $B_{2}^{1}$ and $B_{2}^{2}$ by

$$
B_{2}^{1}=\left\{y \in B_{2}:\left|N_{G^{\prime}}(y) \cap A\right|=1\right\} \quad \text { and } \quad B_{2}^{2}=\left\{y \in B_{2}:\left|N_{G^{\prime}}(y) \cap A\right|>1\right\} .
$$

For another set $\hat{A} \subseteq X$, we denote the sets associated with $\hat{A}$ as $\hat{A}_{0}, \hat{B}_{0}$, etc.

The characterization in the Ore-Ryser Theorem for extendibility to 2-factors in bipartite graphs can now be restated as follows:

Theorem 2. If $H$ is a 2-bounded factor in a regular $(X, Y)$-bigraph $G$, then $H$ extends to a 2-factor in $G$ if and only if for every $A \subseteq X$,

$$
\left|A_{1}\right|+2\left|A_{2}\right| \leqslant\left|B_{1}\right|+\left|B_{2}^{1}\right|+2\left|B_{2}^{2}\right|
$$

From this characterization we derive properties of a minimal 2-bounded factor $H$ that fails to extend to a 2 -factor in $G$. These properties remain requirements when we restrict our attention to matchings in the next section; in that special case we obtain further requirements. The notion of a minimal factor that does not extend is well defined, because if $H$ extends to a 2-factor, then every subgraph of $H$ extends to a 2-factor.

Theorem 3. Let $G$ be a regular $n$-vertex bipartite graph, and let $H$ be a minimal 2bounded factor in $G$ that fails to extend to a 2-factor. Let $A$ be a minimal subset of $X$ whose associated sets violate (*). Let $G^{\prime}=G-E(H)$. The sets associated with A satisfy the following:

(a) $A_{0}=A_{1}=\varnothing$, and every edge of $H$ is incident to $B_{0} \cup B_{1}$.

(b) $2\left|A_{2}\right|=1+\left|B_{1}\right|+\left|B_{2}^{1}\right|+2\left|B_{2}^{2}\right|$.

(c) In $G^{\prime}$, every vertex of $B_{1}$ has at least two neighbors in $A_{2}$. 
(d) Vertices of $A_{2}$ have at most one neighbor in $B_{2}$ that has at most two neighbors in $A_{2}$. Consequently, vertices of $A_{2}$ have at most one neighbor in $B_{2}^{1}$.

(e) $\left|A_{2}\right| \leqslant n / 2-\frac{1}{2}\left(\left|B_{1}\right|+2\left|B_{0}\right|\right)$.

Proof. Consider $x y \in E(H)$. By the minimality of $H$, the sets associated with $A$ in $H-x y$ satisfy $(*)$. We show that this requires (a) through (c).

(a): Deleting $x y$ from $H$ moves $x y$ into $G^{\prime}$. If $x \notin A$ and $y \notin N_{G^{\prime}}(A)$, then this does not change the sets associated with $A$, since $y$ does not move into $N_{G^{\prime}}(A)$. Thus $\left(^{*}\right)$ would also fail for $A$ in $H-x y$. We conclude that every edge of $H$ is incident with $A$ or $N_{G^{\prime}}(A)$. Also no edge of $H$ is incident with $A_{2} \cup B_{2}$, by definition, so $x \in A_{0} \cup A_{1}$ or $y \in B_{0} \cup B_{1}$.

Satisfying $\left(^{*}\right)$ in $H-x y$ requires the right side to increase or the left side to decrease compared to $H$. Consider the right side. Location of a vertex in the partition of $N_{G^{\prime}}(A)$ changes only for the vertex $y$. Possibly $y$ enters $N_{G^{\prime}}(A)$ if $x \in A_{0} \cup A_{1}$; this requires that $y$ has no other neighbor in $A$, so $y$ enters $B_{1} \cup B_{2}^{1}$, and the right side increases by 1 . If already $y \in N_{G^{\prime}}(A)$, then $y$ now needs one additional edge, so $y$ moves from $B_{j}$ to $B_{j+1}$ for some $j$ with $j \leqslant 1$, and the right side increases by at most 1 .

If $x \in A_{i}$ with $i \leqslant 1$, then removing $x y$ moves $x$ from $A_{i}$ to $A_{i+1}$, adding 1 to the left side of $(*)$. Since the right side gains most $1,\left(^{*}\right)$ cannot become satisfied. Hence no edge of $H$ is incident to $A_{0} \cup A_{1}$, which by definition yields $A_{0} \cup A_{1}=\varnothing$, and then $y \in B_{0} \cup B_{1}$.

(b) and (c): By (a), $x \notin A$, and the left side of $\left(^{*}\right)$ does not change when $x y$ moves from $H$ to $G^{\prime}$. Hence the right side must increase by 1 . Since $\left(^{*}\right)$ fails for $H$, it fails by exactly 1, which yields (b). Furthermore, if $y \in B_{1}$, then moving $x y$ from $H$ to $G^{\prime}$ increases the right side only if $y$ moves to $B_{2}^{2}$ and thus has at least two neighbors in $A$.

(d) Now we use the minimality of $A$, without deleting an edge of $H$. Since $(*)$ holds when $A=\varnothing$, we may choose $x \in A_{2}$. Let $r$ be the number of neighbors of $x$ in $B_{2}^{2}$ having exactly one other neighbor in $A_{2}$, and let $s=\left|N_{G^{\prime}}(x) \cap B_{2}^{1}\right|$. Let $\hat{A}=A-x$, so $\left|\hat{A}_{2}\right|=\left|A_{2}\right|-1$.

By (c), in $G^{\prime}$ each vertex of $B_{1}$ has at least two neighbors in $A_{2}$. Thus each vertex of $B_{1}$ has a neighbor in $A_{2}-x$, which yields $B_{1} \subseteq N_{G^{\prime}}\left(\hat{A}_{2}\right)$. Since $H$ has not changed, these vertices still need one incident edge, so $\hat{B}_{1}=B_{1}$. Also $r$ and $s$ have been defined to measure the changes when $A$ is replaced with $\hat{A}$; we have $\left|\hat{B}_{2}^{2}\right|=\left|B_{2}^{2}\right|-r$ and $\left|\hat{B}_{2}^{1}\right|=\left|B_{2}^{1}\right|+(r-s)$. By the minimality of $A$,

$$
2\left|\hat{A}_{2}\right| \leqslant\left|\hat{B}_{1}\right|+2\left|\hat{B}_{2}^{2}\right|+\left|\hat{B}_{2}^{1}\right|
$$

Rewritten in terms of $A$, this becomes

$$
2\left(\left|A_{2}\right|-1\right) \leqslant\left|B_{1}\right|+2\left(\left|B_{2}^{2}\right|-r\right)+\left(\left|B_{2}^{1}\right|+r-s\right) .
$$

Using (b) to cancel the sets in the partition of $A \cup B$ simplifies the inequality to $r+s \leqslant 1$, which is the claim in $(\mathrm{d})$.

(e) For $j \leqslant 1$, every vertex $y \in B_{1}$ has $2-j$ incident edges in $H$ whose other endpoints are in $X-A_{2}$. Each vertex in $X-A_{2}$ can absorb at most two of these edges, since $H$ is 2-bounded. Thus $2\left|X-A_{2}\right| \geqslant\left|B_{1}\right|+2\left|B_{0}\right|$. With $|X|=n / 2$, we have $\left|A_{2}\right| \leqslant n / 2-\frac{1}{2}\left(\left|B_{1}\right|+2\left|B_{0}\right|\right)$. 


\section{Extending Matchings to 2-Factors}

It is well known (by induction on $d$ ) that every edge in a $d$-regular bipartite graph lies in a perfect matching. Similar arguments extend any matching of size 2 to a 2 -factor.

Proposition 4. For $d \geqslant 2$, in a d-regular bipartite graph $G$ every matching with at most two edges extends to a 2-factor.

Proof. Every regular bipartite graph decomposes into perfect matchings. If in such a decomposition the matchings containing the two specified edges are different, then their union is the desired 2-factor. If they lie in the same matching $M$, then the union of $M$ with any perfect matching in the $(d-1)$-regular bipartite graph $G-M$ suffices.

It is somewhat surprising that this easy result is sharp.

Proposition 5. For $d>2$, there exists a d-regular bipartite graph with a matching of size 3 that does not extend to a 2-factor.

Proof. Construct $G$ from two disjoint copies of $K_{d-1, d}$ by adding a matching $M$ joining the partite sets of size $d$. A 2-factor $F$ of $G$ has $4 d-2$ edges. The $2 d-2$ vertices of the smaller partite sets in the two copies of $K_{d-1, d}$ are incident to a total of $4 d-4$ edges in $F$. Thus $F$ contains at most two edges of $M$, and any matching consisting of three edges from $M$ does not extend to a 2 -factor in $G$.

Thus to guarantee extension of matchings to 2-factors in regular bipartite graphs, we must place restrictions on the host graph $G$. Every matching is a 2-bounded factor, so the characterization of extendibility in Theorem 2 applies. When $H$ is a matching, the sets $A_{0}$ and $B_{0}$ associated with $A$ are automatically empty, since each vertex is the endpoint of at most one edge in $H$. Hence the associated sets are just $A_{1}, A_{2}, B_{1}, B_{2}, B_{2}^{1}, B_{2}^{2}$. Statements (a) through (e) in the next theorem specialize the corresponding statement in Theorem 3 , except that (e) here strengthens (e) there.

For a matching $M$, let $U_{M}$ denote the set of endpoints of edges in $M$.

Theorem 6. In a regular n-vertex bipartite graph $G$, let $M$ be a minimal matching that does not extend to a 2-factor. If $A \subseteq X$ is a minimal set whose associated sets violate (*) for $M$, then the following hold:

(a) $A_{1}=\varnothing$, and every edge of $M$ is incident to $B_{1}$ (and hence $B_{1}=U_{M} \cap Y$ ).

(b) $2\left|A_{2}\right|=1+\left|B_{1}\right|+\left|B_{2}^{1}\right|+2\left|B_{2}^{2}\right|$.

(c) In $G-M$, every vertex of $B_{1}$ has at least two neighbors in $A_{2}$.

(d) Vertices of $A_{2}$ have at most one neighbor in $B_{2}$ that has at most two neighbors in $A_{2}$.

(e) $\left|A_{2}\right|=|A| \leqslant n / 2-\left|B_{1}\right|$. 
(f) $2\left|A_{2}\right|>\left|N_{G-M}\left(A_{2}\right)\right|$.

(g) Any subset $U$ of $U_{M} \cap X$ is incident to at least $|U|+1$ edges in $G$ whose endpoints in $Y$ are outside $B_{1} \cup B_{2}^{2}$.

(h) The subgraph of $G-M$ induced by $U_{M}$ has at most $\frac{d}{2}(|M|-1)-|M|$ edges.

Proof. Since a minimal matching that does not extend to a 2-factor is a minimal 2bounded subgraph that does not so extend, statements (a) through (d) were proved in Theorem 3.

(e) Each vertex of $B_{1}$ is incident to one edge of $M$, and edges of $M$ are not incident to $A$. Each vertex outside $A$ is incident to at most one such edge, since $M$ is a matching.

(f) This follows immediately from (b) and $N_{G-M}\left(A_{2}\right)=N_{G-M}(A)=B$.

(g) Let $U=\left\{u_{1}, \ldots, u_{s}\right\}$, with $u_{i} y_{i} \in M$ for $i \in[s]$. Let $\hat{M}=M-\left\{y_{i} u_{i}: i \in[s]\right\}$ and $\hat{A}=A \cup U$. By the minimality of $M$, the sets associated with $\hat{A}$ in $G-\hat{M}$ satisfy $\left({ }^{*}\right)$. Adding $U$ to $A$ increases the left side of $\left(^{*}\right)$ by $2 s$, since $U \subseteq \hat{A}_{2}$. By part (c), each $y_{i}$ moves from $B_{1}$ to $\hat{B}_{2}^{2}$, which increases the right side of $\left(^{*}\right)$ by $s$. Other vertices of $B_{1} \cup B_{2}^{2}$ have the same position in the partition of $\hat{B}$. Vertices outside $B_{1} \cup B_{2}^{2}$ must increase their contribution to the right side of $(*)$ by at least $s+1$. Each edge from $U$ to such a vertex increases the contribution by at most 1 , either by moving a vertex from $Y-N(A)$ to $\hat{B}_{2}^{1}$ or from $B_{2}^{1}$ to $\hat{B}_{2}^{2}$. Hence there must be at least $s+1$ such edges.

(h) Let $t$ be the number of edges of $G-M$ induced by $U_{M}$, and let $m=|M|$. By part (a), $\left|B_{1}\right|=m$. Exactly $d\left|A_{2}\right|$ edges join $A$ to $B$. Among these, exactly $\left|B_{2}^{1}\right|$ are incident to $B_{2}^{1}$, and at most $(d-1) m-t$ are incident to $B_{1}$. Thus $d\left|A_{2}\right| \leqslant(d-1) m-t+\left|B_{2}^{1}\right|+d\left|B_{2}^{2}\right|$. Combining this with part (b) yields

$$
\frac{1}{2}\left(m+1+\left|B_{2}^{1}\right|\right)+\left|B_{2}^{2}\right| \leqslant \frac{d-1}{d} m-\frac{1}{d} t+\frac{1}{d}\left|B_{2}^{1}\right|+\left|B_{2}^{2}\right| .
$$

This inequality simplifies to $(d-2)\left|B_{2}^{1}\right|+2 t \leqslant(d-2) m-d$. Since $\left|B_{2}^{1}\right| \geqslant 0$, we have $t \leqslant \frac{d}{2}(m-1)-m$.

We prove some consequences of Theorem 6. For 3-regular graphs, the content of the first corollary is that all matchings extend to 2 -factors if matchings whose vertices induce no additional edges extend to 2 -factors.

Corollary 7. Let $G$ be a k-regular bipartite graph, where $k \in\{3,4\}$. Every matching in $G$ extends to a 2-factor if and only if every matching $M$ in $G$ with $D_{M} \leqslant k-2$ extends to a 2-factor, where $D_{M}$ is the maximum degree of the subgraph of $G$ induced by $U_{M}$.

Proof. If some matching fails to extend, then choose $M$ and $A$ whose associated sets satisfy the properties in Theorem 6. By part (a), $B_{1}=U_{M} \cap Y$.

If $G$ is 3-regular, then parts (a) and (c) imply that the vertices incident to $M$ induce no edge other than $M$, so $D_{M}=1$.

If $G$ is 4-regular, then part (c) implies that no vertex in $B_{1}$ has more than two neighbors in $U_{M} \cap X$. Part (g) states that every vertex in $U_{M} \cap X$ has at least 2 neighbors outside 
$B_{1} \cup B_{2}^{2}$. Hence these vertices also have degree at most 2 in the subgraph induced by $U_{M}$.

Theorem 6 also restricts the size of the smallest matching in a regular bipartite graph that does not extend to a 2 -factor.

Corollary 8. Let $G$ be a regular n-vertex bipartite graph. If every matching in $G$ with fewer than $n / 3$ edges extends to a 2 -factor, then every matching in $G$ extends to a 2 -factor.

Proof. Let $M$ be a smallest matching that fails to extend to a 2 -factor in $G$. There exists $A \subseteq X$ satisfying the properties obtained in Theorem 6. In particular, $|A|=\left|A_{2}\right|$ and $\left|B_{1}\right|=|M|$, so $|A| \leqslant n / 2-|M|$ and $2|A|=|M|+2\left|B_{2}^{2}\right|+\left|B_{2}^{1}\right|+1$. Thus

$$
n \geqslant 3|M|+2\left|B_{2}^{2}\right|+\left|B_{2}^{1}\right|+1 \geqslant 3|M|+1
$$

and hence $|M|<n / 3$.

The bound of Corollary 8 is sharp when $n=10$. The graph for $d=3$ in Proposition 5 has 10 vertices and has a matching of size 3 that does not extend to a 2 -factor.

\section{Extending Matchings in $Q_{5}$ to 2-Factors}

Although it is unknown whether all matchings in the hypercube extend to 2-factors, we can prove this for the 5-cube, using Theorem 6 and an extended version of a result by Somani and Peleg [18]. We proved this extension in [21] and include a proof here for completeness. (Somani and Peleg considered only the case $|S| \leqslant d+1$, and in this range the conclusion is sharp.) Recall that $N(S)=\bigcup_{v \in S} N(v)-S$; the exclusion of $S$ makes a difference now that we consider sets not confined to one partite set.

Lemma 9. If $S \subseteq V\left(Q_{d}\right)$ with $S \neq \varnothing$, then $|N(S)|>d|S|-\left(\begin{array}{c}|S|+1 \\ 2\end{array}\right)$.

Proof. We use induction on $d$. If $|S|=1$, then $|N(S)|=d>d|S|-\left(\begin{array}{c}|S|+1 \\ 2\end{array}\right)$. When $d=1$ and $|S|=2$, we have $|N(S)|=0>d|S|-\left(\begin{array}{c}|S|+1 \\ 2\end{array}\right)$. Hence we may assume $|S|>1$ and $d>1$.

Since $S$ has at least two vertices, we can split $Q_{d}$ into $(d-1)$-dimensional hypercubes $Q^{1}$ and $Q^{2}$ that each contain some vertex of $S$. Let $S_{i}=S \cap V\left(Q^{i}\right)$. By the induction hypothesis, $\left|N_{Q^{i}}\left(S_{i}\right)\right| \geqslant(d-1)\left|S_{i}\right|-\left(\begin{array}{c}\left|S_{i}\right|+1 \\ 2\end{array}\right)+1$. These neighborhoods are disjoint, so

$$
|N(S)| \geqslant(d-1)\left(\left|S_{1}\right|+\left|S_{2}\right|\right)-\left[\left(\begin{array}{c}
\left|S_{1}\right|+1 \\
2
\end{array}\right)+\left(\begin{array}{c}
\left|S_{2}\right|+1 \\
2
\end{array}\right)\right]+2 .
$$

With $\left|S_{1}\right|+\left|S_{2}\right|$ fixed, $\left(\begin{array}{c}\left|S_{1}\right|+1 \\ 2\end{array}\right)+\left(\begin{array}{c}\left|S_{2}\right|+1 \\ 2\end{array}\right)$ is maximized by maximizing ||$S_{1}|-| S_{2}||$. Thus

$$
|N(S)| \geqslant d|S|-|S|-\left[\left(\begin{array}{c}
(|S|-1)+1 \\
2
\end{array}\right)+\left(\begin{array}{c}
1+1 \\
2
\end{array}\right)\right]+2=d|S|-\left(\begin{array}{c}
|S|+1 \\
2
\end{array}\right)+1 .
$$

Lemma 9 guarantees 11 neighbors for a 6 -vertex set $S$ in $Q_{5}$ contained in one partite set (by the theorem, a 5-vertex subset $S^{\prime}$ of $S$ has 11 neighbors, and $|N(S)| \geqslant\left|N\left(S^{\prime}\right)\right|$ when $S$ is in one partite set). However, we will need one more neighbor. 
Corollary 10. Any six vertices from one partite set in $Q_{5}$ have at least 12 neighbors.

Proof. Otherwise, let $S$ be such a set having only 11 neighbors. Let $X$ and $Y$ be the partite sets, with $S \subseteq X$. Let $T=Y-N(S)$, so $|T|=5$. By Lemma $9,|N(T)| \geqslant 11$. By definition, $N(T)$ and $S$ are disjoint subsets of $X$, but $|S|+|N(T)| \geqslant 6+11>|X|$.

For $d \leqslant 4$, every matching in $Q_{d}$ extends to a spanning cycle and hence to a 2 -factor. Here we extend the latter conclusion to $d=5$.

Theorem 11. Every matching in $Q_{5}$ extends to a 2-factor.

Proof. By Corollary 8, we need only consider matchings with at most 10 edges. Dvořák [4] that linear forests in $Q_{d}$ having at most $2 d-3$ edges extend to spanning cycles; since matchings are linear forests and spanning cycles are 2-factors. matchings with at most 7 edges extend. It remains to consider matchings with 8,9 , or 10 edges. If some matching fails to extend, then let $M$ be a smallest such matching, and let $A$ be a subset of partite set $X$ as guaranteed by Theorem 6 . We use the properties listed in the parts of Theorem 6 to eliminate all cases.

By part (a), no edge of $M$ is incident to $A$; each vertex of $A$ needs two additional edges in a 2 -factor containing $M$ (that is, $A=A_{2}$ ). By part (e), $|A| \leqslant 16-|M|$, so $|A| \leqslant 8$, with equality only if $|M|=8$.

Recall that $B=N_{G-M}(A)$, and $B_{1}$ is the set of vertices in $B$ covered by $M$. By part (a), every edge of $M$ is incident with $B_{1}$, so $\left|B_{1}\right|=|M|$. Now $2\left|A_{2}\right|=\left|B_{1}\right|+2\left|B_{2}^{2}\right|+\left|B_{2}^{1}\right|+1$ from part (b) yields $2|A| \geqslant|M|+1$, and hence $|A| \geqslant 5$.

Since no edges of $M$ are incident to $A$, we have $N_{Q_{5}-M}(A)=N_{Q_{5}}(A)$. Hence we can drop the subscript and refer just to $N(A)$. If $|A|=5$, then Lemma 9 yields $|N(A)| \geqslant 11$; if $|A|=6$, then Corollary 10 yields $|N(A)| \geqslant 12$. However, part (f) requires $2|A|>|N(A)|$, contradicting both cases. Hence $|A| \geqslant 7$.

Lemma 9 implies that any set of size at least 3 in $Y$ has at least 10 neighbors in $X$. All neighbors of $Y-N(A)$ are in $X-A$. Since $|X-A| \leqslant 9$, we have $|Y-N(A)| \leqslant 2$, and hence $|N(A)| \geqslant 14$. However, if $|A|=7$, then part (f) requires $|N(A)|<14$.

We are left with $|A|=8$, which as noted before requires $|M|=\left|B_{1}\right|=8$. Hence part (b) yields $2\left|B_{2}^{2}\right|+\left|B_{2}^{1}\right|=7$. Now, part (f) and $|N(A)| \geqslant 14$ require $|N(A)| \in\{14,15\}$.

First we exclude $|N(A)|=14$. Since $2\left|B_{2}^{2}\right|+\left|B_{2}^{1}\right|=7$, parity implies that $\left|B_{2}^{1}\right|$ is odd, so some vertex $y$ of $N(A)$ has one neighbor in $A$ and hence four neighbors in $X-A$. Let $Y-N(A)=\{u, v\}$. Since $|X-A|=8$, the set $\{u, v\}$ has at most eight neighbors. Hence $u$ and $v$ have two common neighbors; they can't have more, so $N(u) \cup N(v)=X-A$. We also have $y$ with four neighbors in $X-A$.

To eliminate this case, we may assume by symmetry that $u=(00000)$ and $v=$ (11000). The vertices of $N(u) \cup N(v)$ are then all five vertices of weight 1 plus $\{(11100),(11010),(11001)\}$. To have four neighbors in this set, $y$ must have weight 2 . Now $y$ has exactly two neighbors of weight 1 , but $v$ is the only vertex of weight 2 having two neighbors of weight 3 in $N(v)$.

Therefore, $|N(A)|=15$. That is, $8+\left|B_{2}^{2}\right|+\left|B_{2}^{1}\right|=15$. Combining this with $2\left|B_{2}^{2}\right|+$ $\left|B_{2}^{1}\right|=7$ yields $\left|B_{2}^{2}\right|=0$ and $\left|B_{2}^{1}\right|=7$. We count edges incident to $X-A$. Each vertex 
of $B_{2}^{1}$ has one neighbor in $A$ and hence four in $X-A$. The vertex in $Y-N(A)$ has five neighbors in $X-A$. Each vertex of $B_{1}$ has at least one neighbor in $X-A$ (its neighbor in $M$ ). Together, we have at least 41 edges incident to $X-A$, but the total degree of $X-A$ is only 40 .

We have eliminated all cases, so every matching extends to a 2-factor.

\section{References}

[1] Akiyama, J. and Kano, M., Factors and factorizations of graphs: Proof techniques in factor theory. Lecture Notes in Mathematics, 2031. Springer, Heidelberg, 2011. xii+353 pp.

[2] Berman, K. A., Proof of a conjecture of Häggkvist on cycles and independent edges, Discrete Math., 46 (1983), 9-13.

[3] Chen, G., Faudree, R. J., Gould, R. J., Jacobson, M. S., Lesniak, L., and Pfender, F. Linear forests and ordered cycles, Discuss. Math. Graph Theory, 24 (2004), 359-372.

[4] Dvořák, T., Hamiltonian cycles with prescribed edges in hypercubes, SIAM J. Discrete Math., 19 (2005), 135-144 (electronic).

[5] Edmonds, J. and Johnson, E. L., Matching, Euler tours and the Chinese postman, Math. Programming, 5 (1973), 88-124.

[6] Fink, J., Perfect matchings extend to Hamilton cycles in hypercubes, J. Combin. Theory Ser. B, 97 (2007), 1074-1076.

[7] Fink, J., Matching graphs of hypercubes and complete bipartite graphs, European J. Combin., 30 (2009), 1624-1629.

[8] Folkman, J. and Fulkerson, D. R., Flows in infinite graphs, J. Combinatorial Theory, 8 (1970), 30-44.

[9] Ford, L. R. and Fulkerson, D. R., Flows in networks, Princeton University Press, (1962), Princeton, N.J..

[10] Häggkvist, R., On F-Hamiltonian graphs, in Graph theory and related topics, (Proc. Conf., Univ. Waterloo, Waterloo, Ont., 1977) 1979.

[11] Häggkvist, R. and Thomassen, C., Circuits through specified edges, Discrete Math., 41 (1982), 29-34.

[12] Hu, Z. and Tian, F. and Wei, B., Long cycles through a linear forest, J. Combin. Theory Ser. B, 82 (2001), 67-80.

[13] Kano, M. and Saito, A., A short proof of Lovász's factor theorem, Memories of Akashi Technological College, 26 (1984), 167-170.

[14] Katerinis, P., Minimum degree of bipartite graphs and the existence of $k$-factors, Graphs Combin., 6 (1990), 253-258.

[15] Kreweras, G., Matchings and Hamiltonian cycles on hypercubes, Bull. Inst. Combin. Appl., 16 (1996), 87-91. 
[16] Ore, O., Graphs and subgraphs, Trans. Amer. Math. Soc., 84 (1957), 109-136.

[17] Ruskey, F. and Savage, C., Hamilton cycles that extend transposition matchings in Cayley graphs of $S_{n}$, SIAM J. Discrete Math., 6 (1993), 152-166.

[18] Somani, A. and Peleg, O., On diagnosability of large fault sets in regular topologybased computer systems, IEEE Trans. on Comp., 45 (1996), 892-903.

[19] Sugiyama, T., Hamiltonian cycles through a linear forest, SUT J. Math., 40 (2004), 103-109.

[20] Tutte, W.T., Graph Factors, Combinatorica, 1 (1981), 79-97.

[21] Vandenbussche, J., and West, D. B. Matching extendability in hypercubes, SIAM J. Discrete Math., 23 (2009), 1539-1547.

[22] Wojda, A. P., Hamiltonian cycles through matchings, Demonstratio Math., 21 (1988), $547-553$. 